\title{
Electroconvulsive Therapy in Severe Depression: Is Amnesia a Problem?
}

\author{
${ }^{1 *}$ Nilesh M Naphade, ${ }^{2}$ Nikhil S Gupta, ${ }^{3}$ Jyoti V Shetty \\ ${ }^{1}$ Professor, ${ }^{2}$ Resident, ${ }^{3}$ Professor\& Head \\ Department of Psychiatry, Bharati Vidyapeeth Deemed University Medical College \& Research Hospital, Pune- \\ 411043
}

\begin{abstract}
Introduction: Depression is the leading global cause of disability and approximately, 350 million people suffer from depression worldwide. Despite the availability of numerous psychopharmacological treatments, evidence indicates that only 60-70\% of persons who tolerate antidepressants will respond to first-line drug therapy for major depressive disorder. Resistance to the antidepressant medication is the main indication for ECT. However, the use of ECT remains controversial due to concerns about memory impairment in persons with depression who receive acute ECT. The present study was planned toevaluate the effectiveness of ECT in severe depression and its effect on retrograde and anterograde amnesia or any change in their level due to ECT.

Materials \& Methods: A total of 30 consecutive patients diagnosed with Severe Depressive Episode as per International Classification of Diseases (ICD-10) Diagnostic Criteria for Research and satisfying the eligibility criteria were taken in the study after informed consent. ECT was administered as per prevailing guidelines by means of a medical model BPE - 2000 giving bidirectional square wave pulse at a frequency of $20-90 \mathrm{~Hz}$. Baseline evaluation by Hamilton Rating Scale for Depressionwhile Autobiographical Memory Interview (AMI) and Wechsler Memory Scale (WMS) - Third India Edition was used for assessment of retrograde and anterograde amnesia following ECT. Post-treatment evaluation was carried out at the end of ECT treatment and at one month after ECT treatment.

Results: The mean age of the study subjects was 38.53 years with $66.7 \%$ females and $33.3 \%$ males. Significant improvement was seen in depressive symptoms after ECT treatment ( $p<0.05)$. Mean Hamilton score at baseline, immediately after ECT and at 1 month follow up were 40.03, 14.32 and 14.89 respectively ( $p<0.01$ and $p-0.19)$. AMI scores were significantly lower at post-treatment $(79.32 \pm 17.93)$ and follow-up $(76.76 \pm 20.88)$ when compared to pre-treatment AMI scores $(100 \pm 0.00)$. A repeated measures ANOVA which compared mean scores for the immediate and delayed Logical Memory Test (WMS) across treatment did not demonstrate any significant effect for ECT.

Conclusion: The observation made in present study strongly suggest the benefits of ECT in reducing and providing sustained relief from the acute symptoms of depression while no changes were observed in participants anterograde memory. However persistent problems with retrograde amnesia can be a cause of concern. The study findings may assist health care providers and potential patients to take a more informed decisions about their care.
\end{abstract}

Keywords: Autobiographical Memory Interview, Electroconvulsive Therapy, Hamilton Rating Scale, Severe Depression, Wechsler Memory Scale - Third India Edition

\section{Introduction}

Depression is the leading global cause of disability and approximately, 350 million people suffer from depression worldwide [1]. About $13 \%$ of men and $21 \%$ of women will be affected in their lifetime and, according to WHO, Major Depression will be the world's second most debilitating disease by 2020 [1]. India has about 16.6 million cases of Major Depression per year and 10.3 million cases at any time [2].

Despite the availability of numerous psychopharmacological treatments, evidence indicates that only 60-70\% of persons who tolerate antidepressants will respond to first-line drug therapy for major depressive disorder (MDD) [3]. Furthermore, at least one-third of persons with MDD who receive drug therapy will become treatment resistant [4]. Patients who do not have their symptoms fully remitted show higher recurrence risk, anxiety disorders, chronic medical illnesses, have worse psychosocial functioning, worse quality of life (QOL) and higher number of symptoms during follow-up [5-7].

Electroconvulsive therapy (ECT) is a procedure which consists of the induction of convulsive crises by means of applying an electric current through the brain for therapeutic purposes. Resistance to the antidepressant medication is the main indication for ECT [8-11]. Besides, ECT provides a faster response as compared to medication, which is necessary in severe situations that need emergency intervention, such as catatonia and suicide risk [12]. The American Psychiatric Association has already included these situations as 
indication of ECT as the first choice [13].The efficacy of ECT to treat depressive symptoms has been established by means of innumerable studies developed during the last decades [14]. According to Prudic, ECT is the most effective biological treatment currently available for depression, and no other treatment up to now has shown to be superior to ECT in the treatment of major depression in controlled studies [15].

However, the use of ECT remains controversial due to concerns about temporary cognitive impairment in persons with depression who receive acute ECT [16]. Indeed, retrograde and anterograde memory deficits are among the more reliably reported cognitive changes due to ECT $[17,18]$.

The present study was thus planned to evaluate the effectiveness of ECT in severe depression and its effect on retrograde and anterograde amnesia or any change in their level due to ECT, as not much work has been done in this regard in India.

\section{Materials And Methods}

A Prospective Interventional study was conducted in patients with Severe Depression receiving Electroconvulsive Therapy during Oct' 2015 to Sept' 16 . A total of 30 consecutive patients diagnosed with Severe Depressive Episode as per International Classification of Diseases (ICD-10) Diagnostic Criteria for Research and satisfying the eligibility criteria were taken in the study after informed consent [19].

\section{Inclusion Criteria}

1) All patients above 18 years of age.

2) All OPD and IPD patients diagnosed with Severe Depressive Episode (as per ICD-10) receiving Electroconvulsive Therapy.

3) All patients willing to participate in the study by means of Informed consent.

4) All patients giving Informed consent to undergo Electroconvulsive Therapy prior to every treatment.

\section{Exclusion Criteria}

1) All patients who had received Electroconvulsive Therapy within past one year.

2) All individuals with Neurological deficits, serious medical illnesses and Mental Retardation.

3) All individuals with other Psychiatric and Neurological disorders like Dementia, Mania, Psychosis, and Substance use disorders.

\section{Methodology}

A written Informed Consent after explaining the procedure in detail was taken before each ECT treatment. Baseline evaluation was done one day prior to ECT treatment while Post-treatment evaluation was carried out at the end of ECT treatment and at one month after ECT treatment.All the observations were recorded on a specially designed proforma and validated by two experienced psychiatrists independently.

\section{Depression assessment}

The Hamilton Rating Scale for Depression (HAM-D) [20] was used to assess depressive symptomatology in all participants at pre-treatment, post-treatment, and follow-up testing sessions. This study utilized the 21-item interview which rates the severity of symptoms observed in depression such as low mood, insomnia, agitation, anxiety and weight loss. The questionnaire is one of the most commonly used scales for rating depression in medical research. The investigator rates the patient by interviewing them and observing the patient's symptoms. Each question has from three to five possible responses that increase in severity. The total score represents a sum of the values for the questions. Scores ranging 0-7 were normal, from 8 to 13 indicate mild depression symptomatology, 14 to 18 indicate moderate depression, 19 to 22 indicate severe depression and scores above 22 are interpreted as indicating very severe depression.

\section{Retrograde Memory assessment}

Retrograde amnesia was assessed with the Autobiographical Memory Interview - Short Form (AMI, Kopelman MD, Wilson BA, 1989) [21]. This instrument was developed specifically for the purpose of examining the nature of amnesia following ECT, and allows the quantification of retrograde amnesia following ECT treatment. To accomplish this, the participant was asked a series of detailed autobiographical questions prior to ECT. The questions probed for specific details for six sets of events or experiences, most of which occurred during the previous year. The six domains inquired in detail about the participant's most recent employment, birthday celebration, New Year's Eve activities, information about a close relative, recent travel, and most recent physical complaint for which they sought medical care. Each of these six domains contained six specific questions (i.e., Where did you celebrate your last birthday?). These same questions were asked again at post-treatment and follow-up. Thus, the participant's answers prior to treatment were used as a templateagainst which subsequent answers were compared. The comparison of the post-treatment answers with the answers 
given prior to treatment yielded "amnesia scores" in the form of percentiles. Individual norms have not been developed for this instrument, yet it remains the primary outcome measure for personally relevant autobiographical memory currently utilized in ECT research.

\section{Anterograde amnesia assessment}

Wechsler Memory Scale - Third India Edition (WMS-III) [22] is specially designed Observer Rating Scale to assess Anterograde amnesia; Memory indexes such as immediate recall, delayed recall, logical memory and working memory for both verbal and visual domains. The administration time per individual is around 75 minutes.We used the WMS-III Logical Memory subtests to assess auditory recall under immediate and delayed conditions. In the immediate condition (Logical Memory I) the participant listened to two stories and retold the details of the stories over three trials, one trial for story 1 and two trials for story 2 . The participants' raw score for all three trials was summed and converted to a subtest scaled score $(M=10, S D=3)$. After a 25- to 35minute delay (Logical Memory II), the participant was asked to retell the details of the two stories. In this case, the raw scores from the two trials were summed and converted to scaled scores. The split-half reliability coefficients, providing measures of internal consistency, were reported in the manual for both subtests (Wechsler, 1997b). Averaged across all age groups, the reliability coefficient for Logical Memory I was 0.88 and for Logical Memory II was 0.79 , indicating high internal consistency. Test-retest reliability coefficients, providing estimates of the subtest stability over time, were also reported. Averaged across all age groups, the test-retest reliabilities for Logical Memory I and Logical Memory II were 0.77 and 0.76 , indicating moderately high stability over time.

\section{Electroconvulsive Therapy}

ECT was administered as per prevailing guidelines by means of a medical model BPE - 2000 giving bidirectional square wave pulse at a frequency of $20-90 \mathrm{~Hz}$ [23]. Seizure threshold was determined by the half age method of Petride and Fink [24].

\section{Statistical Analysis}

All the collected data was entered in Microsoft Excel Sheet 2007.The data was then transferred and analyzed using SPSS ver. 17. Qualitative data was represented in the form of frequency and percentage and analyzed by chi-square test while quantitative data was represented using mean +/- S.D and analyzed using repeated measure ANOVA with post-hoc Bonferroni test. A p-value of $<0.05$ was taken as level of significance.

\section{Results}

The mean age of the study subjects was 38.53 years with $66.7 \%$ females and $33.3 \%$ males. Most of the subjects had between 2-4 episodes during their course of illness. Other psychiatric morbidities were seen in $40 \%$ of the cases (Table 1). Immediately post-treatment 6 participants had HAM-D scores in the range indicating no depression (score < 8), 17 had scores in the mild to moderate depressed range (scores 8-18), 7 demonstrated severe/ very severe depression $(>/=19)$. These data indicate that the majority of the participants responded to ECT treatment. At 1-month follow-up, 4 patients fell in the range indicating no depressive symptoms, 24 in the mild to moderate range and 2 in the severe/ very severe range. Mean Hamilton score was 40.03, 14.32 and 14.89, before ECT, immediately after ECT and at 1 month follow up respectively ( $<<0.01$ and $p-0.19$ ) (Table 2 and 3). Results of the ANOVA demonstrated that AMI scores differed significantly across the three test administrations $(\mathrm{p}<0.01)$. Post-hoc comparisons analyses using the Bonferroni adjustment for significance indicated that the average AMI scores were significantly lower at post-treatment (79.32 \pm 17.93$)$ and follow-up $(76.76 \pm 20.88)$ when compared to pre-treatment AMI scores $(100 \pm 0.00)$, yielding statistically significant results for both comparisons $(\mathrm{p}<0.05)$ (Table 4).Mean scores for the immediate and delayed Logical Memory Test (WMS) across treatment did not demonstrate a significant change after ECT i.e. there were no statistically significant changes in participants' immediate or delayed recall between pre and post-treatment and follow-up testing (Table 5).

\section{Discussion}

Among the most vexing predicaments for many clinicians is providing dispassionate guidance regarding electroconvulsive therapy (ECT) to a patient and family members of a severely impaired, suicidal, medication-resistant patient, who has benefited marginally from psychotherapy. The American Psychiatric Association (2001) regards it as a very effective option when other treatments fail [1]. Present hospital based prospective observational study was conducted to evaluate changes in depressive symptoms and their sustenance in patients with severe depression. In present study, all the 30 subjects had severe/very severe depression before ECT therapy. Immediately post-treatment 6 participants had HAM-D scores in the range indicating no depression, 17 had scores in the mild to moderate depressed range, 7 demonstrated severe/ very severe 
depression. Our findings indicate that the majority of the participants responded to ECT treatment. At 1-month follow-up, 4 patients fell in the range indicating no depressive symptoms, 24 in the mild to moderate range and 2 in the severe/ very severe range. Mean Hamilton score was 40.03, 14.32 and 14.89, before ECT, immediately after ECT and at 1 month follow up respectively ( $<<0.01$ and $\mathrm{p}-0.19)$. These findings shows that ECT provided sustained relief from symptoms of depression.

In results of the study by Saccoman et al. [25] indicate that ECT is an effective treatment of severe depression. Their study showed that $88 \%$ of the participants responded immediately after completion of a standard series of bifrontal ECT. Our success rate is also comparable to the 70 to $90 \%$ response rate for all types of ECT reported by the American Psychiatric Association's (2010) practice guidelines regarding treatment for depression [26], and higher than remission rates of 55\% and $64 \%$ found in multisite research consortium projects $[27,28]$.

Like other investigations, the current study also found that few participants experienced a return of depressive symptoms 1 month after treatment cessation and that on average participants demonstrated an increasing trend in depression symptomatology from post-treatment to follow-up. Specifically, $6.5 \%$ of the participants who were classified as 'no depression' as per Hamilton scale were later classified as 'mild depression' after the cessation of treatment. Saccoman et al. [25] found the relapse rate of $13.5 \%$ in their study. Relapse rate in our study is very low as compared to some other studies of ECT (such as Prudic et al. [15], who reported a 64\% 24-week relapse rate in their 2004 publication and Grunhaus et al. [29], who reported a 28.5\% 3month relapse rate in their 2001 study).

Very low relapse rates at follow-up in our study may be due to the lack of a longer follow-up period. This is considered a limitation of this study. The present data are restricted to only 1 month follow-up which is much shorter than the follow-up periods of 24 weeks from Prudic et al. [15] and 3 months in Grunhaus et al. [29] This provides reason to be cautious in making strong conclusions about ECT relapse rates. It is plausible that given the trend in the current sample of rising symptomatology longer follow-up periods may have resulted in a higher relapse rate. But still, ECT can be considered an effective course of action for severe, medicationresistant, depressive conditions.

Memory loss and confusion represent the bulk of the complaints from individuals who have received ECT treatment [30,31]. Very few studies (Kellner et al., 2010; Lawson et al., 1990) had attempted to assess memory functions in ECT with targeted neuropsychological measures [27,32]. The present findings failed to corroborate the findings of Kellner et al. [27] concerning ill effects of ECT on measures of verbal anterograde memory. While Saccoman et al. [25] found no evidence of impaired anterograde memory on any of the measures administered, suggesting that ECT did not result in impaired recall of newly learned information [104]. Further, contrary to the findings of Kellner et al. [27], current results did not suggest impairment in immediate or delayed recall of information in learning a list of words. While in study of Saccoman et al. [25], results of immediate and delayed tests of list learning were suggestive of average improvement at follow-up testing over pre- and post-treatment performance. A possible interpretation of these results is that the relief of depression resulted in improved verbal memory functions by follow-up, while an alternative consideration is that the improvement is attributable to practice effects from repeated exposure to the list of words.

Unfortunately, like previous ECT findings (Kellner et al. [27]; Sackeim et al., [28], Saccoman et al. [25]) the current study showed persistent problems with retrograde amnesia. Whether retrograde amnesia will persist beyond 1 month is unclear; however, data from other investigations, including that of Sackeim et al. [28], suggest that by 6 months post-treatment, there may be substantial improvement in the ability to remember autobiographical information. In present study, the results of the Autobiographical Memory Interview revealed that bitemporal ECT had a significant impact on the accuracy of participants' memories of previously recalled personally relevant information. On average, participants remembered only $79.32 \%$ of the information provided at pre-treatment interviews immediately following the completion of their series of ECT treatments. At 1-month follow-up they remembered $76.76 \%$. Contrary to findings that ECT does not produce cognitive effects that persist after treatment cessation [32] and the hypothesis that bitemporal ECT may spare memory functions [33] these results suggest that memory of personally relevant biographical information is significantly compromised with bitemporal ECT treatment and persists at least 1 month post-treatment.

These findings are consistent with those of Kellner et al. [27] and Saccoman et al. [25] who demonstrated that ECT caused impairment on this measure. Kellner et al. [27] found that participants who received ECT could remember only $69 \%$ of the information provided on the AMI at baseline, whereas Saccoman et al. [25] found an average recall on the AMI of $72 \%$ immediately post-treatment. Therefore, the current results suggest slightly less impairment in retrograde amnesia as compared to Kellner et al. [27] and Saccoman et al. [25].

Again, a lack of longer follow-up testing presents a limitation in interpreting this study's retrograde amnesia data. It remains unknown whether retrograde amnesia would persist indefinitely or would dissipate after more time. Another limitation is that the AMI represents an imperfect measure of retrograde amnesia. For 
example, if participants offer inaccurate or vague memories during the pre-treatment interview, that can confound their accuracy of recall at post-treatment and follow-up. Even though participants were encouraged to offer only clear and accurate memories, there was no mechanism to check accuracy. Since depression itself is believed to negatively affect memory and cognition [34], pre-treatment responses could already be compromised by participants' depressed states. The accuracy of the measure could be improved by consulting with participants' families and friends to corroborate baseline responses, although if the individual has encoded the memory inaccurately, efforts to corroborate responses may confound the issue further. That said, the AMI is the most widely used measure of retrograde memory employed in ECT research. It likely represents the only standardized approach to measuring the autobiographical memory loss, which has long been a complaint of those who have completed ECT treatment [35].

\section{Conclusion}

These results help clarify to a great extent what patients can expect to experience following a treatment with ECT. In weighing the benefits versus the risks, these data strongly suggest the benefits are substantial in reducing the acute symptoms of depression while sparing Anterograde memory. However persistent problems with retrograde amnesia can be a cause of concern. The study findings will help patients to understand what the risks are concerning cognitive side effects of ECT and may assist health care providers and potential patients to take a more informed decisions about their care. Providing this knowledge upfront may serve to greatly reduce an individual's anxiety and fear prior to ECT treatment. Subsequently these results may help reduce the stigma of ECT, making it more likely to be utilized as a treatment option for severe depression.

\section{References}

[1]. The World Health Organization. The global burden of disease: 2004 update, table A2: burden of disease in DALYs by cause, sex and income group in WHO regions, estimates for 2004. Geneva, Switzerland: WHO, 2008. http://www.who.int/healthinfo/global_ burden_disease/GBD_report_2004update_AnnexA.pdf (accessed 6 Mar 2014)

[2]. S Saxena, A Dhawan. Disability and Burden of depressive disorders. The National Medical Journal of India; vol.12,No.2,1999

[3]. Souery D, Papakostas GI, Trivedi MH. Treatment resistant depression. J Clin Psychiatry 2006;67(Suppl 6):16-22.

[4]. Fava M, Davidson KG. Definition and epidemiology of treatmentresistant depression. Psychiatr Clin North Am 1996;19:179-200.

[5]. Kennedy N, Foy K. The impact of residual symptoms on outcome of major depression. Curr Psychiatry Rep. 2005;7(6):441-6.

[6]. Rush AJ, Kraemer HC, Sackeim HA, Fava M, Trivedi MH, Frank E, Ninan PT, Thase ME, Gelenberg AJ, Kupfer DJ, Regier DA, Rosenbaum JF, Ray O, Schatzberg AF, ACNP Task Force. Report by the ACNP task force on response and remission in major depressive disorder. Neuropsychopharmacology. 2006;31(9):1841-53.

[7]. Viinamaki H, Hintikka J, Tolmunen T, Honkalampi K, Haatainen K, Koivumaa-Honkanen H. Partial remission indicates poor functioning and a high level of psychiatric symptoms: a 3-phase 6-year follow-up study on major depression. Nord J Psychiatry. 2008;62(6):437-43.

[8]. Husain SS, Kevan IM, Linnell R Scott AI. Electroconvulsive therapy in depressive illness that has not responded to drug treatment. J Affect Disord. 2004;83(2-3):121-6.

[9]. Freeman C, Hendry J, Fergusson G. National audit of electroconvulsive therapy in scotland; 2000. Available from: http://www.sean.org.uk.

[10]. Braga RJ, Petrides G. Somatic therapies for treatment-resistant psychiatric disorders. Rev Bras Psiquiatr. 2007;29(Suppl 2):S77-84.

[11]. Husain SS, Kevan IM, Linnell R, Scott AI. What do psychiatrists mean by medication resistance as an indication for electroconvulsive therapy? J ECT. 2005;21(4):211-3.

[12]. McCall WV. What Does Star*D Tell Us About ECT? J ECT. 2007;23(1):1-2.

[13]. American Psychiatric Association Task Force on Electroconvulsive Therapy. The practice of electroconvulsive therapy: recommendations for treatment, training and privileging. Washington, DC: American Psychiatric Association; 2001.

[14]. Abrams R. Electroconvulsive therapy. 4th edn. Oxford, UK: Oxford University Press, 2002.

[15]. Prudic J. Electroconvulsive therapy. In: Sadock BJ, Sadock VA, editors. Kaplan \& Sadock's comprehensive textbook of psychiatry. 8th ed. Philadelphia: Lippincott Williams \& Wilkins; 2005. p.2968-83.

[16]. Lisanby SH. Electroconvulsive therapy for depression. N Engl J Med 2007;357:1939-45.

[17]. UK ECT Review Group. Efficacy and safety of electroconvulsive therapy in depressive disorders: a systematic review and metaanalysis. Lancet 2003;361:799-808.

[18]. Semkovska M, McLoughlin DM. Objective cognitive performance associated with electroconvulsive therapy for depression: a systematic review and meta-analysis. Biol Psychiatry 2010;68:568-77.

[19]. World Health Organization. The ICD-10 classification of mental and behavioural disorders: clinical descriptions and diagnostic guidelines. Geneva: World Health Organization; 1992.

[20]. Hamilton M. A rating scale for depression. Journal of Neurology, Neurosurgery \& Psychiatry. 1960 Feb 1;23(1):56-62.

[21]. Kopelman MD, Wilson BA, Baddeley AD. The autobiographical memory interview: a new assessment of autobiographical and personal semantic memory in amnesic patients. Journal of Clinical and Experimental Neuropsychology. 1989 Oct 1;11(5):724-44.

[22]. Wechsler D. Wechsler memory scale (WMS-III). San Antonio, TX: Psychological Corporation; 1997. Adaptation and Standardization carried out byDr Pushpalatha Gurappa (NIMHANS, Banglore).

[23]. Davidson JR. Major depressive disorder treatment guidelines in America and Europe. The Journal of clinical psychiatry. 2009 Dec;71:04-17.

[24]. Petrides G, Fink M. The" half-age" stimulation strategy for ECT dosing. The Journal of ECT. 1996 Sep 1;12(3):138-46.

[25]. Saccoman ML. An investigation of the cognitive effects of electroconvulsive therapy in the treatment of severe depression (Doctoral dissertation, The University of Utah).

[26]. American Psychiatric Association. (2010). Practice guideline for the treatment of patients with major depressive disorder (3rd Ed.) American Psychiatric Association: Arlington, VA. 
[27]. Kellner CH, Knapp RG, Petrides G, et al. Continuation electroconvulsive therapy vs pharmacotherapy for relapse prevention in major depression: a multisite study from the Consortium for Research in Electroconvulsive Therapy (CORE). Arch Gen Psychiatry 2006; 63:1337.

[28]. Sackeim HA, Dillingham EM, Prudic J, et al. Effect of concomitant pharmacotherapy on electroconvulsive therapy outcomes: short-term efficacy and adverse effects. Arch Gen Psychiatry 2009; 66:729.

[29]. Grunhaus L, Shipley JE, Eiser A, Pande AC, Tandon R, Remen A, Greden JF. Shortened REM latency PostECT is associated with rapid recurrence of depressive symptomatology. Biological psychiatry. 1994 Aug 15;36(4):214-22.

[30]. Malcolm, K. (1989). Patients' perceptions and knowledge of electroconvulsive therapy. Psychiatric Bulletin, 13,161-165.

[31]. Cronholm B, Ottosson JO. The experience of memory function after electroconvulsive therapy. The British Journal of Psychiatry. 1963 Mar 1;109 (459):251-6.

[32]. Squire LR, Slater PC, Chase PM. Anterograde amnesia following ECT. Behavioral biology 1976;17:31-41.

[33]. Inglis, J. (1969). Electrode placement and the effect of E.C.T. on mood and memory in depression. Canadian Psychiatric Association Journal, 14, 463471.

[34]. Calev, A., Gaudino, E.A., Squires, N.K., Zervas, I.M., \& Fink, M. (1995). ECT and non-memory cognition: A review. British Journal of Clinical Psychology, $34,505-515$.

[35]. Rose, D., Wykes, T., Leese, M., et al (2003) Patients' perspectives on electroconvulsive therapy: systematic review. BMJ, 326, 1363- 1367.

\section{TABLES}

Table 1. Distribution of patients according Baseline Characteristics

\begin{tabular}{|l|l|l|}
\hline Baseline Characteristics (n-30) & N & \% \\
\hline Variables & & \\
\hline Age group (yrs) & 2 & $6.7 \%$ \\
\hline$</=30$ & 16 & $53.3 \%$ \\
\hline $31-40$ & 11 & $36.7 \%$ \\
\hline $41-50$ & 1 & $3.3 \%$ \\
\hline$>50$ & & \\
\hline Sex & 20 & $66.7 \%$ \\
\hline Female & 10 & $33.3 \%$ \\
\hline Male & & \\
\hline Family History of Psychiatric Illness & 7 & $23.3 \%$ \\
\hline No & 23 & $76.7 \%$ \\
\hline Yes & & \\
\hline Course of Present Illness & 22 & $73.3 \%$ \\
\hline Gradual & 8 & $26.7 \%$ \\
\hline Sudden & & \\
\hline Medical Co-morbidity & & $63.3 \%$ \\
\hline No & 19 & $36.7 \%$ \\
\hline Yes & 11 & \\
\hline No. of Episodes & & $13.3 \%$ \\
\hline 1 & 4 & $83.3 \%$ \\
\hline $2-4$ & 25 & $3.3 \%$ \\
\hline$>4$ & 1 & $60.0 \%$ \\
\hline Psychiatric Co-morbidity & & $70.0 \%$ \\
\hline No & 18 & $73.3 \%$ \\
\hline Yes & 12 & $26.7 \%$ \\
\hline Substance Abuse & & \\
\hline No & 22 & 8 \\
\hline Yes & 8 & \\
\hline & & \\
\hline
\end{tabular}

Table 2. Distribution of subjects as per severity of depression

\begin{tabular}{|l|l|l|l|l|l|l|}
\hline \multirow{2}{*}{ Hamilton Score } & Baseline & Immediate After ECT & \multicolumn{1}{l|}{ 1 month F/u } \\
\cline { 2 - 7 } & $\mathbf{N}$ & $\mathbf{\%}$ & $\mathbf{N}$ & $\mathbf{\%}$ & $\mathbf{N}$ & \% \\
\hline No Depression & 0 & $0.0 \%$ & 6 & $20.0 \%$ & 4 & $13.3 \%$ \\
\hline Mild & 0 & $0.0 \%$ & 17 & $56.7 \%$ & 19 & $63.3 \%$ \\
\hline Moderate & 0 & $0.0 \%$ & 6 & $20.0 \%$ & 5 & $16.7 \%$ \\
\hline Severe & 30 & $100.0 \%$ & 1 & $3.3 \%$ & 2 & $6.7 \%$ \\
\hline Total & 30 & $100.0 \%$ & 30 & $100.0 \%$ & 30 & $100.0 \%$ \\
\hline p- value & $\mathbf{0 . 0 1}$ & & $\mathbf{0 . 7 1}$ \\
\hline
\end{tabular}


Table 3. Distribution of subjects based on mean change in Hamilton score

\begin{tabular}{|l|l|l|l|}
\hline Hamilton Scale & Mean & SD & p- value \\
\hline Baseline & 40.03 & 8.76 & \multirow{2}{*}{} \\
\hline Immediately after ECT & 14.32 & 9.62 & $\mathbf{0 . 1 9}$ \\
\hline 1 month After ECT & 14.89 & 9.54 & $\mathbf{0 . 1 9}$ \\
\hline
\end{tabular}

Table 4. Distribution of subjects based on Mean change in Autobiographic Memory Interview Score

\begin{tabular}{|l|l|l|l|}
\hline AMI & Mean & SD & p-value \\
\hline Baseline & 100.00 & 0.00 & \multirow{2}{*}{$<\mathbf{0 . 0 1}$} \\
\hline Immediately after ECT & 79.32 & 17.93 & $\mathbf{0 . 1 3}$ \\
\hline 1 month After ECT & 76.76 & 20.88 & \\
\hline
\end{tabular}

Table 5. Distribution of subjects based on change in Wechsler Memory Scale -III score WMS- III

\begin{tabular}{|c|c|c|c|}
\hline Logical Memory I & Mean & SD & p-value \\
\hline Baseline & 9.25 & 2.57 & \multirow{3}{*}{0.17} \\
\hline Immediately after ECT & 8.81 & 3.60 & \\
\hline 1 month After ECT & 9.69 & 3.01 & \\
\hline Logical Memory II & Mean & SD & p-value \\
\hline Baseline & 10.40 & 2.67 & \multirow{3}{*}{0.22} \\
\hline Immediately after ECT & 9.23 & 4.99 & \\
\hline 1 month After ECT & 10.60 & 2.13 & \\
\hline
\end{tabular}

